\title{
MALWARE DETECTION USING HONEYPOT AND MALWARE PREVENTION
}

\author{
Dhruvi Vadaviya \\ Student, Cyber Security, GTU School of Engineering \& Technology, \\ Ahmedabad, Gujarat, India \\ Mahesh Panchal \\ Deputy Director, Gujarat Technological University, Ahmedabad, Gujarat, India \\ Dr. Abdul Jhummarwala \\ BISAG, Gandhinagar, Gujarat, India
}

Dr. M. B. Potdar

Project Director, BISAG, Gandhinagar, Gujarat, India

\begin{abstract}
The main intention of this paper is to elaborately discuss about seriousness of Malware problem and project the importance. of online malware analysis in Malware defense research. Reaching the security of network systems is one of the most essential and popular technology in the area of information technology. One of the methods applied for network security is the use of honeypots. Network safety includes recording and analysis of network activities and captures, to find out the proof about the source of the attacks to the device safety.
\end{abstract}

Key words: Terms - Honeypot, Low-level interaction, Threshold resource value, High-level interaction, Medium level interaction, Honeyd, Intrusion detection, Network security, Malware Detection, Malware Prevention

Cite this Article: Dhruvi Vadaviya, Mahesh Panchal, Dr. Abdul Jhummarwala, Dr. M. B. Potdar, Malware Detection Using Honeypot and Malware Prevention, International Journal of Computer Engineering and Technology 10(6), 2019, pp. 1-9. http://iaeme.com/Home/issue/IJCET?Volume=10\&Issue $=6$

\section{INTRODUCTION}

Honeypots are a research tool in the field of information security and have proven their value as a powerful educational tool in modern classrooms. Many public and private researchers and organizations who are part of the information security community are currently using a trap-style network to learn the tactics, techniques, and procedures that the hacker community is using to infiltrate the information store without authorization, which can contain sensitive information. 
Your system may contain fake data that may be of interest to hackers. By accessing data, hackers can disclose identifying information such as IP address, geographic location, computer platform, and other data. This information can be used to enhance security for hackers and similar users.

Another example of a honeypot is an email honeypot designed to prevent spammers. It can consist of a fake e-mail address that is deliberately added to a list of known spam. Email addresses can be used to track servers and relays that send spam to your honeypot account.

This information can be used to block specific IP addresses and domain names in the antispam database. Email honeypots are also available as a counter-attack tool to forward spammers to the email address of the identified spammers.

Honeypots are computer security mechanisms that attempt to detect, mislead, or otherwise interfere with attempts to exploit an information system.

In general, honeypots consist of data that appears to be a legitimate part of the site, but is actually quarantined and monitored (such as network sites), and appears to contain information or valuable resources to the attacker. It was blocked at that time. This is similar to a detention facility known as "baiting" as a suspect.

Honeypot also provides teachers and students with tools to thoroughly analyze security events in a modular fashion, a very desirable feature in teaching information security courses.

However, honeypot technology is rapidly evolving, and honeypots are difficult to ignore in a year or two. New developments will evolve lab technology with familiar names for fullblown enterprise-class security tools.

\section{RELATED BACKGROUND}

\subsection{Types of Honeypot}

There are many types of honeypots. The level of interaction and the purpose of the honeypot. There are three types depending on the level of interaction. There are two types of honeypot depending on the purpose. We can see everything in detail as below.

\subsubsection{Based on Level of Interaction}

The most common classification types are based on the level of interaction provided by a honeypot to a malicious user. The more interactive the environment, the closer the honeypot is to the actual attack target, the more accurate information can be collected. The disadvantage is that more realistic honeypots are more difficult to configure and configure. There are three levels:

\section{Low-Interaction}

- You can use one or more simple services that record all communication attempts to a specific service, such as a web or SSH server.

- Typically, these are simple daemons that provide people with a passive method of monitoring attack attempts.

- Because host operating systems are not vulnerable to attack, honeypots with low interaction are fairly safe to run, but cannot be used where a more complex and interactive environment is required, such as an SMTP server.

\section{Medium-Interaction}

- Medium-level honeypots begin to emulate a collection of software to provide more compelling information to attackers, but still protect the host operating system. 
- While emulated programs should respond in the same way as real programs, emulating a collection of software can be quite complicated because there should not be the same security issues. Otherwise, the emulation will be aborted.

- It also increases the risk of system corruption due to more attack points for malicious users.

\section{High-Interaction}

- Finally, there is a large interaction honeypot. The entire host operating system is presented to the attacker with an actual instance of the program instead of an emulation.

- A common goal of an interactive honeypot is to determine what an attacker is doing after obtaining root access from the system.

- Because of this goal, this level of honeypot is the most dangerous but has the greatest potential to gather information.

- This level of honeypot requires constant oversight because the attacker will actually control it and can use the attack as a jump point for additional attacks.

Table 1 Tradeoffs of Honeypot Levels of Interaction

\begin{tabular}{|l|l|l|l|l|}
\hline $\begin{array}{c}\text { Level of } \\
\text { Interaction }\end{array}$ & $\begin{array}{c}\text { Work to Install } \\
\text { and Configure }\end{array}$ & $\begin{array}{c}\text { Work to } \\
\text { Deploy and } \\
\text { Maintain }\end{array}$ & $\begin{array}{c}\text { Information } \\
\text { Gathering }\end{array}$ & \multicolumn{1}{|c|}{$\begin{array}{c}\text { Level of } \\
\text { Risk }\end{array}$} \\
\hline Low & Easy & Easy & Limited & Low \\
\hline Medium & Involved & Involved & Variable & Medium \\
\hline High & Difficult & Difficult & Extensive & High \\
\hline
\end{tabular}

\subsection{Motives of Attackers}

Recognizing the motives of an attacker can be a great help in understanding the threats. To help you understand the motivational issues, the Department used MICE abbreviations for money, ideology, compromise (which you did not want to do) and the self. Social group admission and status. Here are some of the many reasons why an attacker could try to break into the system as a target. Almost all these motivations have been confirmed using honeypots.

- Denial of Service: A DoS attack is an attack designed to remove a victim's computer system or network. This is typically done by populating the intended destination (such as a web server) with network traffic. The more traffic the victim receives, the more effective the attack. Attackers attack thousands (if not thousands) of systems that will be used to attack. The more computers you own, the more traffic you can run on your destination. Many blackhats use other DoS attacks to remove other blackhats. The IRC war is an example of an individual attempting to knock someone out on an IRC channel using a DoS attack.

- BOTs: BOTsare automated robot that work on behalf of an individual in a preprogrammed way. They are most commonly used to maintain IRC control. As more computers are hacked, more BOTs can be fired and more IRC channels can be controlled. With many BOTs, individuals will not be able to control IRC from denial of service (DoS) attacks.

- Credit Cards: A hacked computer has become a form of call. Blackhats will replace the hacked account with a stolen credit card. The more computers are hacked; the more money you can make. This behavior is documented in the Honeynet Project's "Know Your Enemy: Motives" article.

- Bragging Rights: Many Black Hat organizations have the ability: Your position is based on your strengths and your skills. To increase your status, you must demonstrate your skill. Often you need to break into another site. The more sites you enter, the more you get. We often see 
people who modify websites, damaging the system, to be proud of their skills and try to improve their position.

- CPU Cycles: Some worms were developed to replace the CPU cycles of the client system to win the contest. The more computers the worm is infected with, the more the combined CPU cycles are used. The more machines and processing power the attacker handles, the greater the chance of winning the contest.

- Corporate Espionage: An organization may attempt to breach a competitor's security in order to gain a competitive advantage. This is a common motive for advanced blackhats because it involves financial interests.

\subsection{Advantages of Honeypots}

There are many security solutions on the market. Anyone can navigate through the Internet and find the solution that best suits their needs. Here are some reasons to choose a honeypot:

- Honeypots can capture additional information about the attack by capturing the attack and providing information from the attack type and, if necessary, the log.

- You can see new attacks and create new security solutions.

- If you look at the type of malicious behavior, you can get more tests. It can help you understand more attacks that can occur.

- Honeypots are not bulky in terms of data capture. They only handle incoming malicious traffic. Therefore, the information captured is not as much as the total traffic. Focusing only on malicious traffic makes it much easier to investigate. Therefore, honeypot is very useful.

- The only malicious traffic does not require large data storage. No need to keep up with new technology. All computers can be used as honeypot systems. So, there is no additional budget to create such a system.

- They are easy to understand, configure, and install. They do not have complex algorithms. You do not need to update or change some things.

\subsection{Disadvantages of Honeypots}

Honeypots also have some disadvantages because there are some important advantages of using honeypots.

- You can capture data only if the hacker actively attacks the system. If he does not attack the system, he cannot catch information. If an attack occurs on another system, the honeypot cannot identify it. This can damage the system other than the attack on the honeypot system and cause big problems.

- Honeypot fingerprint has a disadvantage. It is easy to understand whether a skilled hacker is attacking a honeypot system or an actual system. Fingerprinting allows you to distinguish between the two. It is not the result of our experiments.

- Honeypots can be used as zombies to reach other systems and damage your system. This can be very dangerous.

\subsection{Honeypot Deployment Strategies}

After implementation and testing, the honeypot must be deployed to the existing network. As needed, honeypots can be deployed in a variety of strategies and locations on the network. There are various legal issues with honeypot deployment technology. 


\subsection{Sacrificial Lamb}

- This is the simplest of all deployment technologies. As its name suggests, honeypots are on a network that is waiting for attackers to attack.

- That is, while the attacker provides what he wants and keeps track of it while keeping it busy

- Honeypots are not always production-worthy, but they help ensure that managers have time to act.

Examples of honeypots that can be deployed using the Sacrificial lamb strategy include Deception tool kit (DTK) and Specter.

\subsection{Deception Ports on Production Systems}

- This deployment strategy mimics the various services on different ports of the system. For example, HTTP imitates on port 80 and SMTP imitates on port 25.

- The idea here is very similar to the idea of scapegoat technology. That is, an attacker can fool an attacker in such a way that they can take actions such as tracers, tricks, and so on while they are trying to crack the trick.

- This kind of deployment is the most common and least responsive. Examples of honeypots that can be deployed using this strategy include Honeyd and Specter.

\subsection{Proximity Decoys}

- The key idea of this deployment strategy is to keep the honeypot close to the production server.

- If you determine that the honeypot is on the same subnet as the one on which the primary server resides, it means that it is technically on the same network.

- Therefore, there is no legal hassle because you have the right to use technology to protect one asset in the network.

- It will also be easier to reroute or trap attacks targeting production servers.

- For example, you deploy a honeypot using VMware and User mode Linux (UML).

\subsection{Redirection Shield}

- Honeypots deployed using this strategy primarily use port redirection or traffic rerouting.

- This strategy is one of the most promising strategies because it has the most commercial value.

- Honeypots can be used as services, not devices, just like Software as a Service (SaaS).

- This strategy is primarily concerned with installing a rerouting switch at the client site.

- Honeypots can now sit anywhere in the world to receive all traffic coming into the server and track hackers.

\subsection{The Core Technology of Honeypots}

As part of intrusion detection systems, honeypots are used primarily in network spoofing techniques in all defense systems, collecting information based on data, and controlling intruder access and other technologies. Key technologies of honeypot: data control, data collection, data analysis. 8920 


\subsection{Internet Spoofing Technology}

- Honeypot, we can also call it bait attack system. It only reflects the value of the attacker.

- The main role of network spoofing technology is to require intruders to spend a lot of time and resources to detect the attacker's means of attack, achieve the purpose of the attack, and protect the real network.

- Honeypot technology now includes several implications, including IP address spoofing, simulation system vulnerability attacks, network traffic simulation, and dynamic port configuration of the system.

\subsection{Data Analysis}

- Data analysis is a key function of the honeypot system and the ultimate goal of building a honeypot system is to analyze the data.

- Honeypot system log analysis primarily affects the nature of the attack behavior, which is a difficult problem for the honeypot system, as honeypots collect a lot of information and do not need the necessary connections between the information.

- To better analyze the information, you must create an information analysis module to set up a data analysis model in which the attacker's actions are performed.

\subsection{Data Collection}

- Data collection refers to the honeypot design of the core module, monitoring all activity to be recorded with reference to the honeypot.

- It is difficult for us to collect as many threats as possible throughout the intrusion detection process.

- The more information you capture, the better it is to analyze these attacks and analyze the attacker's motives, strategies, and tools.

\subsection{Data Control}

- Data control can inhibit network intrusions that could allow an attacker to use a honeypot to attack or harm a non-honeypot system.

- Once an attacker enters the honeypot system, every effort should be made to reduce the damage to the non-honeypot system. You can minimize the risk. Different data control technologies and methods have different risks, but they cannot completely eliminate the risk.

\section{PROBLEM DEFINITION}

After reviewing all papers and related background information, we can identify some of the following problems.

The challenge is to simplify the process, improve data analysis, and increase the value of the honeynet, including expanding the dataset used for pre- and post-response IDS analysis.

If you need multiple devices, you can install a control sensor, depending on your network configuration, to monitor and connect the embedded device.

Such a system can develop an embedded system or appliance combined with a Web-based user interface when the system administrator enters minimal information at the deployment stage.

Another problem is to materialize the subprogram command to increase efficiency or to ensure that the user specifies subprogram commands to suit the environment and data collection requirements. Exciting system users can not configure the system.

Increase the scalability and reliability. 


\section{IMPLEMENTATION STRATEGY}

There is limitation that, when data or packet transfer if there are any changes in the packets during transformation it's not detect, because its low-level interaction Honeypot.

So, for the solution I prepare the following plan,

In this I create a network topology. It consists 25 - nodes and server. Calculate the energy for all nodes. All Nodes are transfer the initial id and energy level to the server. The server receives the initial id from nodes and calculate threshold resource value. Next the Server send the Resource value to Nodes. Based on the value it compares the initial id and then the resource will be allocated and then perform the packet transmission. So, if there are any changes in the packets its id is different and we can say that intrusion happened in packet transmission.

Step 1 : Create a network topology that consists 25 - nodes and server

Step 2 : Calculate the energy for all nodes

Step 3 : All Nodes are transfer the initial id and energy level to the server

Step 4 : The server receive the initial id from nodes and calculate threshold resource value.

Step 5 : Next the Server send the Resource value to Nodes.

Step 7 : Based on the value the resource will be allocated and then perform the packet transmission.

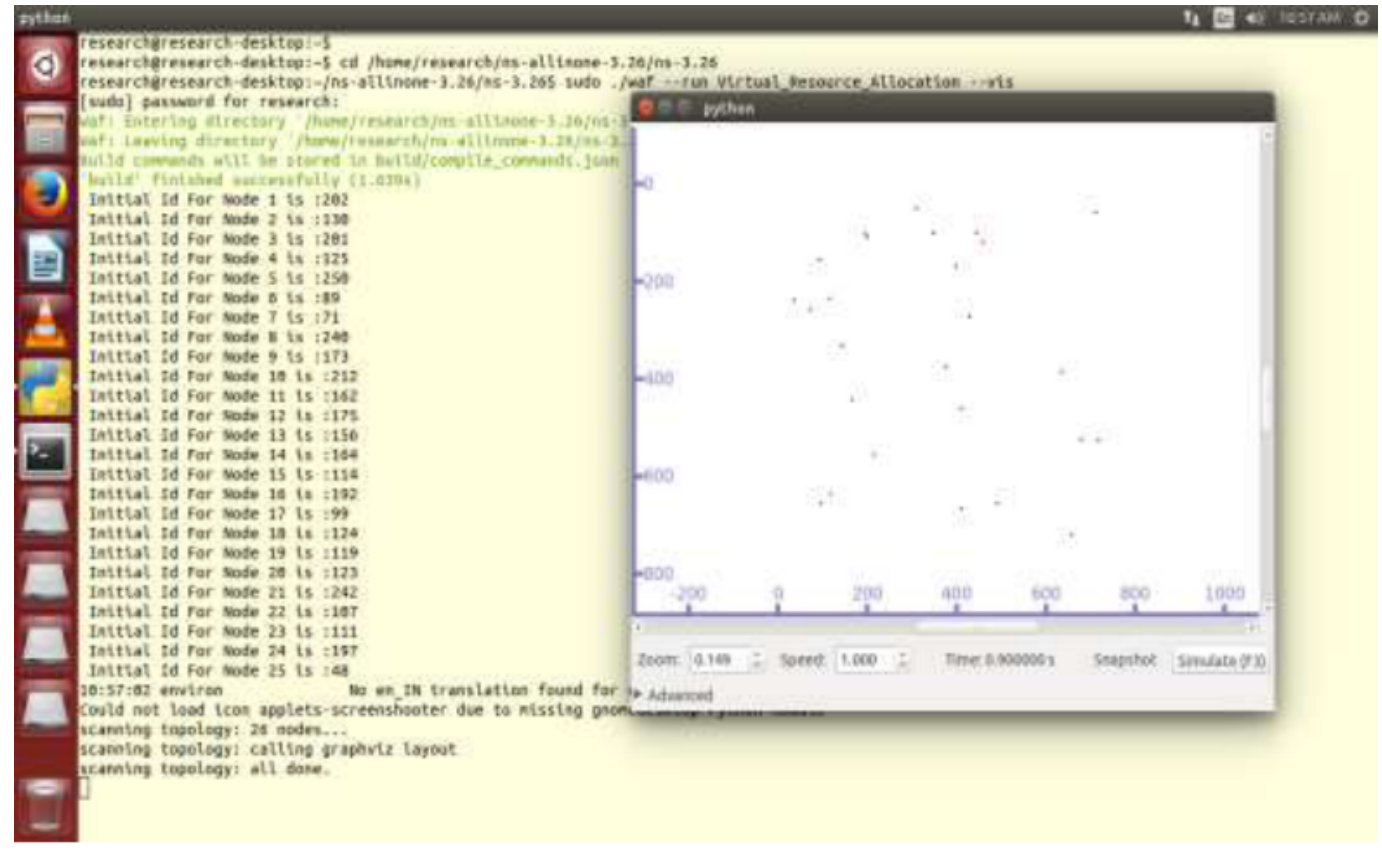

Figure 1 Initial ID for all nodes 
Dhruvi Vadaviya, Mahesh Panchal, Dr. Abdul Jhummarwala, Dr. M. B. Potdar

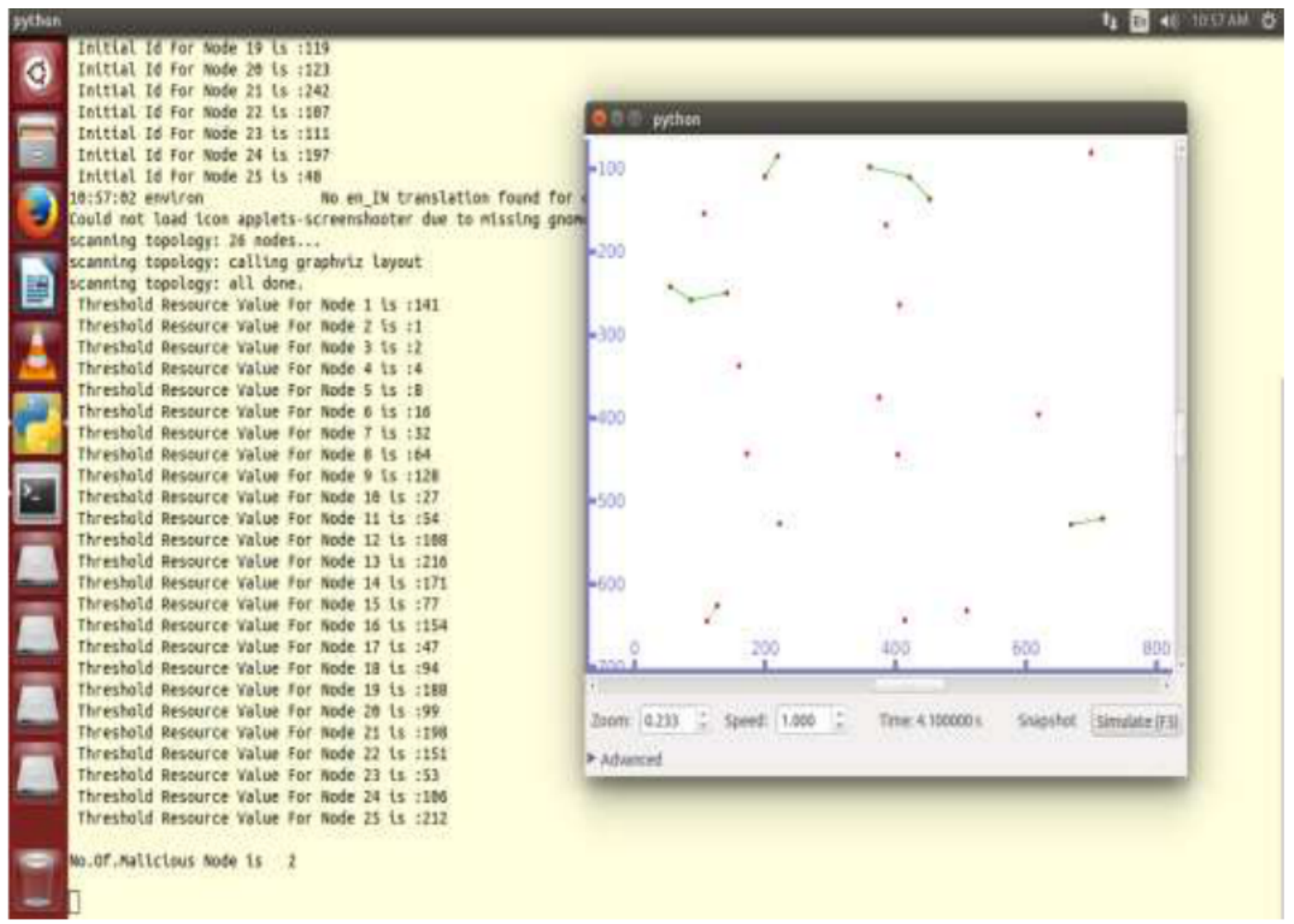

Figure 2 Threshold resource value

As shown in fig. 2 there is threshold value of node 2 is 1 , it means it's had malicious data.

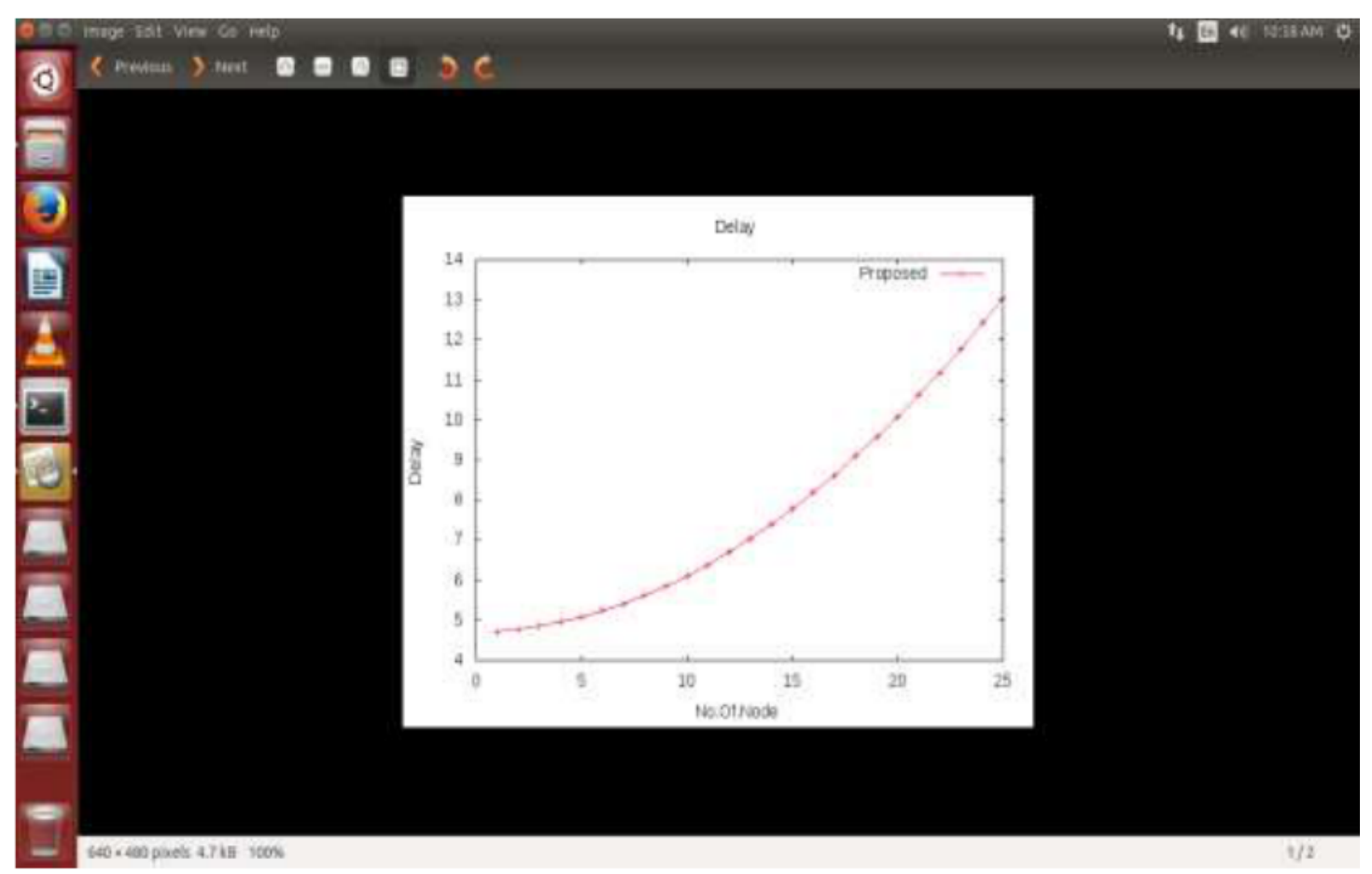

Figure 3 Delay vs. No. of Nodes 


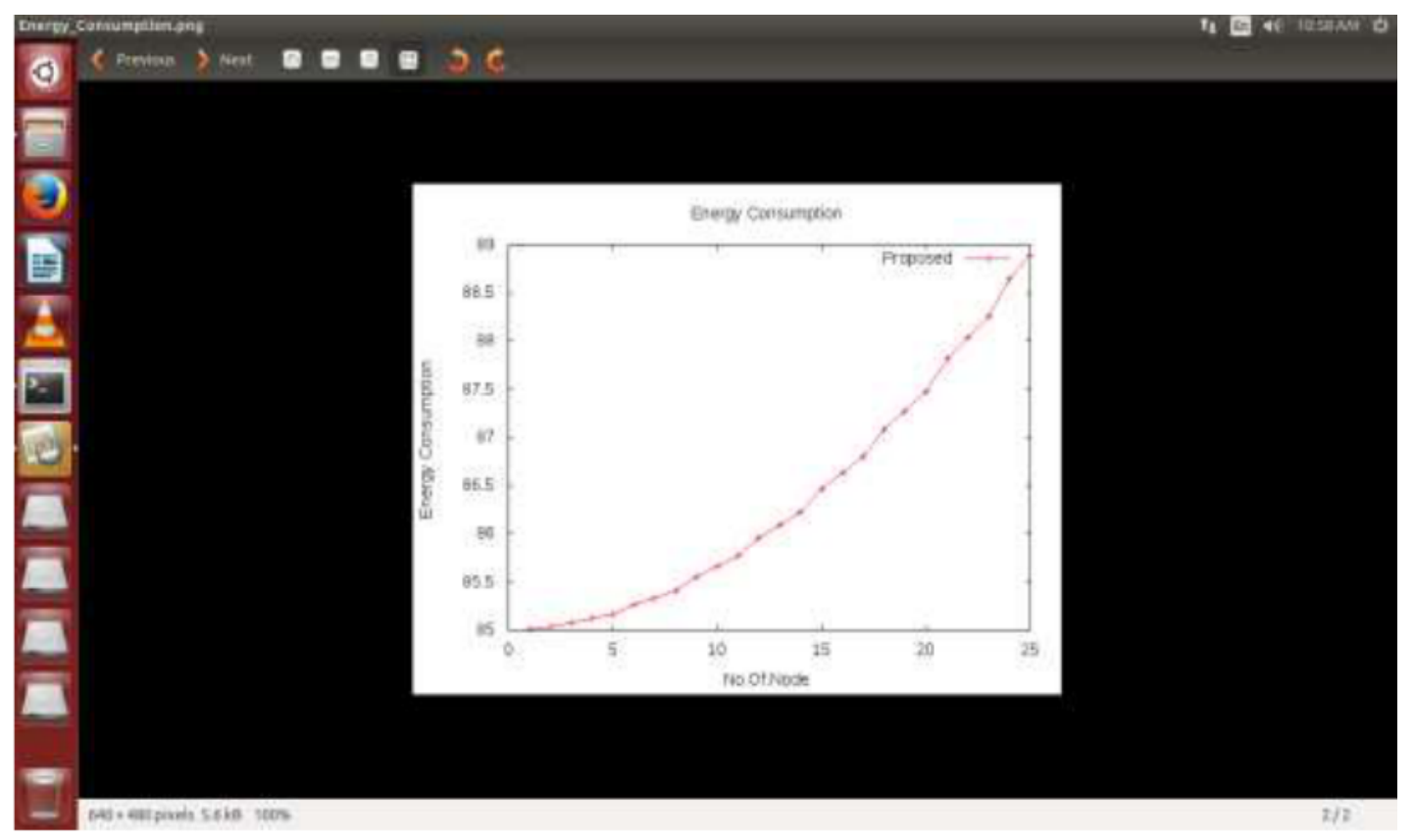

Figure 4 Energy Consumption vs. No. of Nodes

\section{CONCLUSION}

We presented an approach for effective Malware defense by using threshold value. The proposed has been designed by integrating Honeypot technology, Intrusion Detection Systems and malware analysis in Windows based platform. We also discussed about research limitations in Malware defense. In future, it can be employed offline using python based forensic analysis.

\section{REFERENCES}

[1] Anagnostakis, Kostas G., et al. "Detecting Targeted Attacks Using Shadow Honeypots." Usenix Security Symposium. 2005.

[2] Borisaniya, Bhavesh, et al. "Incorporating Honeypot for intrusion detection in cloud infrastructure." IFIP International Conference on Trust Management. Springer, Berlin, Heidelberg, 2012.

[3] Dahbul, R. N., C. Lim, and J. Purnama. "Enhancing Honeypot deception capability through network service fingerprinting." Journal of Physics: Conference Series. Vol. 801. No. 1. IOP Publishing, 2017.

[4] Rodrigues, Marcos, and OlamilekanShobayo. "Design and Implementation of a Low-Cost Low Interaction IDS/IPS System Using Virtual Honeypot Approach." Covenant Journal of Informatics \& Communication Technology 5.1 (2017): 48-64.

[5] Diansyah, TengkuMohd, et al. "Analysis of Using Firewall and Single Honeypot in Training Attack on Wireless Network." Journal of Physics: Conference Series. Vol. 930. No. 1. IOP Publishing, 2017.

[6] Cao, Jianhong, et al. "Dipot: A distributed industrial Honeypot system." International Conference on Smart Computing and Communication. Springer, Cham, 2017.

[7] Singh, Abhay Nath, Shiv Kumar, and R. C. Joshi. "Intrusion Detection System Based on Real Time Rule Accession and Honeypot." International Conference on Network Security and Applications. Springer, Berlin, Heidelberg, 2011.

[8] Tiwari, Ritu, and Abhishek Jain. "Design and analysis of distributed Honeypot system." International Journal of Computer Applications 55.13 (2012). 